\title{
Transformando el Programa Intensivo sobre el Futuro de la Banca y Las Finanzas en un encuentro virtual Transforming the Intensive Programme on the Future of Banking and Finance into an online event
}

\author{
Fernández Sánchez, Pedro ${ }^{1}$, Frank, Elizabeth ${ }^{2}$ \\ fersan.fcee@ceu.es, elizabeth.frank@ceu.es \\ ${ }^{1}$ Departamento de Economía \\ Universidad San Pablo CEU \\ Madrid, España \\ ${ }^{2}$ Departamento de Empresa \\ Universidad San Pablo CEU \\ Madrid, España
}

\begin{abstract}
Resumen- El estallido de la pandemia originado por el virus SARSCOVID 19 ha obligado a un replanteamiento de nuestra vida en todos los ámbitos. Inmersos en este nuevo contexto, y con el objeto de continuar ofreciendo a nuestros estudiantes una formación integral que incluya no sólo conocimientos, sino también competencias, que les permitieran incorporarse a un mercado laboral que también se ha transformado radicalmente, se decidió convertir el Programa Intensivo sobre el Futuro de la Banca y las Finanzas en un evento virtual. Durante seis meses se trabajó en un nuevo planteamiento, que ha permitido a los estudiantes participantes continuar trabajando de manera colaborativa en un entorno multicultural.
\end{abstract}

Palabras clave: Trabajo autónomo, trabajo cooperativo, internacionalización, encuentro virtual, aprendizaje basado en proyectos

Abstract- The outbreak of the pandemic caused by the SARS-COVID 19 virus has forced us to rethink our lives. Immersed in this new context, and with the aim of continuing to offer our students a comprehensive training that includes not only knowledge but also skills that will enable them to join a labour market that will also have to adapt to this new situation in the short term. With this objective in mind, the decision was taken to transform the Intensive Programme on the Future of Banking and Finance into an online event, in which the participating students would continue to work collaboratively in a multicultural environment.

Keywords: Autonomous work, cooperative work, online meeting, internationalization, project-based learning

\section{INTRODUCCIÓN}

En el año 2014 varias universidades europeas pusieron en marcha el Programa Intensivo sobre Ética y Finanzas en Rotterdam. El objetivo era, partiendo de las causas que habían originado la Gran Recesión en 2008, debatir sobre qué había fallado más allá de lo estrictamente económico, así como sobre el papel que había jugado la ética en la toma de decisiones y qué enseñanzas se podrían extraer de cara al futuro. Este Programa Intensivo (en adelante PI), fue visto por la Facultad de Ciencias Económicas y Empresariales de la Universidad San Pablo CEU como un instrumento para fomentar el trabajo autónomo y colaborativo de nuestros estudiantes. Permitía, además, añadir un beneficio adicional para los futuros egresados: la variable internacional. Los alumnos de la USP CEU podrían, además, utilizar el trabajo realizado durante la semana de celebración del PI como base para su Trabajo de Fin de Grado. Se convirtió de esta manera en una apuesta de la Facultad, que desde entonces $\mathrm{y}$ año tras año ha participado de manera activa en el mismo. De hecho, en el año 2015 se encargó de su organización, al celebrarse en Madrid. El año siguiente el encuentro tuvo lugar en Gante (Bélgica), siendo Katowice (Polonia), Praga (república Checa) y Zaragoza las sedes del programa en los años siguientes.

Durante el curso académico 2019-2020 y cuando los estudiantes iban a viajar a Rotterdam a participar en la semana de PI, tuvo lugar el cierre de fronteras para hacer frente a la primera oleada de la epidemia provocada por el virus SARSCOVID 19. Por ello que se procedió a su suspensión. Las Universidades participantes, conscientes de la importancia que tiene este programa en la formación de los estudiantes, al iniciarse el curso 2020-2021 comenzaron a trabajar en un nuevo encuentro. La situación epidemiológica impedía retomar el encuentro físico, por lo que surgió la cuestión ¿por qué no utilizar las herramientas de las que disponíamos y que se habían desarrollado durante el cierre para convertirlo en un encuentro virtual? De esta forma y a lo largo de seis meses se trabajó en el diseño de un nuevo PI virtual, enfocado en reforzar el fomento del trabajo colaborativo entre los estudiantes, que había sido el eje en las ediciones anteriores. Al fin y al cabo el aprendizaje no es más que un proceso acumulativo, autorregulado, dirigido hacia objetivos, de carácter individual y colaborativo (Van den Bergh et al., 2006). Precisamente esta es la filosofía que inspira el PI.

El presente trabajo trata de explicar la conversión de un programa que giraba en torno al encuentro en una sede física cada curso académico, en un evento virtual. Se trata de continuar avanzando en el cambio metodológico basado en promover y tutelar el trabajo autónomo de los estudiantes, así como en el diseño de nuevos programas basados en competencias. Todo ello sin perder de vista el objetivo de crear 
una experiencia para los estudiantes, donde puedan asociar conceptos tales como aprendizaje, participación, trabajo individual y trabajo cooperativo (Fernández Batanero, 2004).

\section{CONTEXTO}

La imposibilidad de realizar el encuentro físicamente planteó a las universidades que cada año participaban en el PI la necesidad de adaptarse a la nueva situación mundial provocada por la irrupción del SARS COVID 19 en nuestras vidas. Tras siete ediciones en las que estudiantes y profesores se trasladaban a una ciudad europea para debatir sobre los temas objeto del encuentro, resultaba necesario, si se quería que los objetivos de éste se continuaran cumpliendo, darle una nueva orientación. El nuevo encuentro no debía olvidar el propósito que habían guiado su puesta en marcha y celebración durante más de un lustro: fomentar el pensamiento crítico sobre el futuro de las finanzas y potenciar el trabajo autónomo y el trabajo en equipo de los estudiantes, todo ello dentro de un entorno internacional. Se trata, por tanto, de un aprendizaje basado en proyectos (o project- based learning), con el que se pretende, a través de un proyecto complejo y significativo que se desarrolla en varias fases, que los estudiantes adquieran determinadas competencias mediante la promoción del trabajo autónomo y colaborativo (García Valcárcel y Basilotta, 2017).

Para diseñar el nuevo PI se contaba además con el desarrollo de herramientas informáticas que habían permitido transformar el trabajo presencial en un trabajo a distancia durante el cierre mundial.

\section{A. Objetivos}

Desde el mes de septiembre de 2020 hasta febrero de 2021 se creó un grupo de trabajo formado por profesores de la Universidad de Inholland (Países Bajos), del University College de Gante (Bélgica), de la Universidad de Katowice en Polonia, de la Universidad de Heilbronn (Alemania), de la Universidad de Administración y Finanzas de Praga (República Checa), de la Universidad de Zaragoza (España), y de la Universidad San Pablo CEU de Madrid (España). A través de reuniones semanales mediante Microsoft Teams, se crearon grupos de trabajo encargados de diseñar el nuevo PI. De esta forma se consiguió convertirlo en un encuentro virtual, incorporando importantes novedades, entre las que cabría destacar el refuerzo del trabajo cooperativo. Resulta necesario recalcar que esta transformación no ha consistido en hacer en línea lo que antes se realizaba de manera presencial. Los cambios han ido mucho más allá, al haberse modificado sustancialmente la dinámica del encuentro para hacerlo más colaborativo. Además, se ha potenciado la interrelación de los diferentes temas en los que se organizaba el encuentro.

\section{B. Público Objetivo}

Estudiantes de los últimos cursos de los grados en Administración y Dirección de Empresas y del Grado en Economía de las Facultades de Ciencias Económicas y empresariales de diferentes universidades europeas. Todos ellos interesados en el ámbito de las finanzas.

\section{DESCRIPCIÓN}

En las siguientes líneas se explicarán las principales novedades introducidas en el PI para el curso académico 20202021. Para ello se analizarán las dos fases en que se divide el
PI: la fase preparatoria y la fase de celebración propiamente dicha del encuentro.

\section{A. La fase preparatoria o el pre-PI}

Quizás es esta fase donde han tenido lugar los cambios más profundos. Esta etapa solía comenzar en el mes de enero del año académico de celebración del encuentro. Una vez seleccionados los estudiantes de cada institución que iban a participar, éstos debían escoger un tema específico de los tratados en el PI:

Tema 1: Rating empresarial

Tema 2: Imposición y regulación fiscal

Tema 3: Gobierno corporativo

Tema 4: El futuro de la banca más allá del horizonte 2020

Tema 5: Sistemas alternativos de financiación de pequeñas y medianas empresas

\section{Tema 6: Inversiones socialmente responsables}

Los procesos de selección varían según las universidades. En el caso de la Universidad San Pablo CEU se tienen en cuenta cuatro aspectos: nota media del expediente académico, nivel de inglés, carta de motivación y ejercicio de dinámica de grupo. De igual manera los sistemas de incentivos varían enormemente en cada institución: a nuestros estudiantes les sirve de base para su TFG, en otras universidades les permiten obtener créditos ECTS, y en otras los alumnos participan porque el tema de encuentro les interesa.

Cada participante, encuadrado en cada uno de los 6 temas y a partir de las pautas fijadas en la guía del estudiante (donde se encuentras fijados los objetivos del tema y la bibliografía), debía responder a una serie de cuestiones. El objetivo de este trabajo previo era tanto que los alumnos adquirieran conocimientos teóricos necesarios sobre el tema objeto de estudio, como que manejaran información sobre la situación específica de su país. Cada tema era liderado por dos profesores, que actuaban como mentores y que guiaban a los estudiantes en sus respuestas, que debían estar subidas en la plataforma una semana antes de la celebración del encuentro. El objetivo de esta fase preparatoria era fomentar el trabajo autónomo de los participantes. Para ayudar a la consecución de este objetivo se creaban grupos de WhatsApp para cada tema, donde los estudiantes debían colgar pequeñas presentaciones, explicaban sus inquietudes y su día a día, y planteaban dudas o preguntas sobre el trabajo individual que debían realizar.

La fase preparatoria del PI virtual ha cambiado sustancialmente en el curso 2020-21, si bien hay aspectos que se mantienen. Los temas se mantienen, pero los estudiantes entre febrero y marzo deben tanto contestar cuestiones sobre su tema específico, como elaborar un artículo en grupo, en base a ese trabajo autónomo apoyado en las cuestiones. En todo momento continúan siendo asesorados por los dos o tres profesores que tutelan el grupo. Se continúan empleando WhatsApp y el correo electrónico como herramientas de apoyo. Además se añade el empleo de la plataforma Microsoft Teams, a través de la cual se realizan reuniones, pueden subir sus documentos individuales y, lo más importante, preparan un documento conjunto de manera colaborativa.

La elaboración de un artículo internacional era lo que se hacía en los encuentros físicos en la etapa pre-covid. Los estudiantes durante la semana de la reunión elaboraban un 
documento académico sobre el tema objeto de estudio. Ahora, en el PI virtual este trabajo se realiza en la fase preparatoria. Los profesores establecen un calendario para cada uno de los temas y los miembros del equipo deben ir contestado las cuestiones planteadas (en eso no hay cambio con respecto a la situación anterior). Como si de un rompecabezas se tratara, el objetivo es que adquieran conocimientos y obtengan información de cara a la elaboración del documento científico. Ese artículo ha de estar finalizado antes del comienzo del encuentro virtual, y es fruto de la colaboración de los estudiantes de cada tema, (siempre bajo la supervisión de sus profesores). Como se ha comentado anteriormente Microsoft Teams permite mediante su sistema de compartir archivos, ir realizando comentarios, sugerencias y proponiendo cambios. En última instancia facilita enormemente el trabajo colaborativo. Ya no es necesario que estudiantes y profesores estén físicamente. Cada uno puede trabajar desde su propia institución/ vivienda y país de residencia.

El principal cambio introducido en esta fase preparatoria del PI ha consistido, por tanto, en hacer a lo largo de la misma lo que anteriormente se realizaba en la fase preparatoria y durante el encuentro. El objetivo: potenciar el trabajo colaborativo, fundamentalmente de cara al encuentro virtual, donde, como se explicará a continuación, los diferentes grupos han de colaborar entre sí.

\section{B. La semana del PI}

Como ya se ha comentado, antes del establecimiento de las restricciones a los movimientos de personas como consecuencia de la pandemia, el PI se desarrollaba presencialmente en una ciudad europea. Durante una semana estudiantes y profesores realizaban una serie de actividades entre las que se encontraban un encuentro multicultural, visitas culturales a instituciones y a la ciudad y una conferencia sobre un tema relacionado con el futuro de la banca y de las finanzas impartida por un profesional de reconocido prestigio. Debían escribir un documento internacional, como se acaba de explicar, y defenderlo a través de una presentación. Los trabajos realizados por los estudiantes, (el escrito y la presentación), eran además calificados por los profesores. Para una información más detallada puede consultarse Fernández Sánchez y Frank (2019)

La transformación del PI en un encuentro virtual ha supuesto modificar por completo esta dinámica. El documento internacional que anteriormente elaboraba cada grupo de estudiantes durante la reunión presencial ahora ya está finalizado (se escribió durante el pre PI). ¿Qué hacen entonces los estudiantes durante los tres días del encuentro virtual? Para potenciar el trabajo en equipo y la colaboración, se unen los temas de dos en dos. El objetivo es que profundicen en sus conocimientos mediante la comparación, la aplicación y la presentación de resultados entre los distintos temas. Los nuevos grupos han de analizar la forma en que ambos temas están interrelacionados y se influyen mutuamente. Los tres grupos, resultantes de la unión por pares de los 6 temas, son:

Grupo 1: en el que trabajan conjuntamente los estudiantes que investigaron sobre los temas 1 y 2 .

Grupo 2: resultado de unir a los estudiantes que trabajaron sobre los temas 3 y 6 .

Grupo 3: en el que colaboran los estudiantes que investigaron sobre los temas 4 y 5
El día de comienzo del PI virtual y con el objeto de que los estudiantes se conozcan entre ellos, ya que sólo han tenido contacto con sus compañeros de tema, se pone en marcha una actividad para que interactúen con los participantes de los otros temas, a los que todavía no conocen. Esta actividad es un escape room intercultural virtual, en el que varios equipos compiten entre ellos por finalizar en primer lugar un juego de pistas y pruebas (y obtener un pequeño premio). Este juego les permite desarrollar habilidades como el trabajo en equipo, idiomas (inglés), capacidad de liderazgo y trabajo bajo presión. Aparte, es imprescindible que el alumno desarrolla una profunda comprensión de los diversos contextos culturales (pues precisamente el juego versaba sobre un recorrido por diferentes enclaves europeos). El éxito de este trabajo en equipo globalizado y virtual requiere habilidades específicas para navegar por las diferencias culturales (Meyer, 2014).

Finalizado este juego virtual, los 3 grupos comienzan a trabajar en la redacción de la adenda. Primero, a través de un video y con formato pecha- kucha, exponen a sus compañeros del otro tema los principales objeticos de su propio tema y las conclusiones que han obtenido al final de la fase preparatoria. De esta forma se inicia el proceso de discusión de las relaciones entre ambos temas y la elaboración de un trabajo académico que se incorporará como adenda. De nuevo la plataforma Teams se convierte en la herramienta que facilita el trabajo colaborativo entre los estudiantes.

Desde el lunes hasta el miércoles y bajo la supervisión de los profesores de los temas "reunidos", cada uno de los tres grupos trabaja para tener preparada la adenda el miércoles por la mañana. El miércoles por la tarde deben exponer al resto de compañeros los principales objetivos de sus trabajos, así como las conclusiones obtenidas. En paralelo durante esta etapa profesores invitados de diferentes instituciones financieras y gubernamentales imparten conferencias. Para ello se establecen horarios para que los estudiantes puedan conectarse a estas clases magistrales relacionadas con los temas objeto de discusión del encuentro.

A modo de resumen en la tabla 1 se pueden observar las actividades planeadas en cada una de las fases del PI, así como los objetivos perseguidos con las mismas, antes de que estallara la pandemia y en la actualidad cuando el PI se ha convertido en un encuentro virtual.

\section{Tabla 1}

Actividades y objetivos del PI presencia y del PI virtual

\begin{tabular}{|c|c|c|}
\hline & $\begin{array}{c}\text { PI antes del } \\
\text { COVID }\end{array}$ & PI virtual \\
\hline & Pre PI & Pre PI \\
\hline 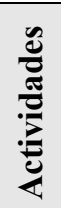 & $\begin{array}{l}\text { Responder a las } \\
\text { cuestiones propias de } \\
\text { cada tema }\end{array}$ & $\begin{array}{l}\text { Responder a las cuestiones } \\
\text { propias de cada tema } \\
\text { Elaboración de un trabajo } \\
\text { científico sobre el tema del } \\
\text { tema }\end{array}$ \\
\hline 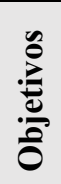 & $\begin{array}{l}\text { Fomento del trabajo } \\
\text { autónomo }\end{array}$ & $\begin{array}{l}\text { Fomento del trabajo } \\
\text { autónomo } \\
\text { Fomento del trabajo } \\
\text { colaborativos }\end{array}$ \\
\hline
\end{tabular}




\begin{tabular}{|c|c|c|}
\hline & Semana del encuentro & Encuentro virtual \\
\hline 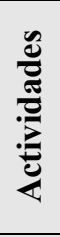 & $\begin{array}{l}\text { Elaboración de un } \\
\text { trabajo científico sobre } \\
\text { el tema del tema } \\
\text { Presentación del trabajo }\end{array}$ & $\begin{array}{l}\text { Presentación del tema } \\
\text { Elaboración de un trabajo } \\
\text { científico conjunto entre } \\
\text { temas } \\
\text { Presentación del trabajo } \\
\text { por grupos }\end{array}$ \\
\hline$\frac{2}{0} \cdot \frac{2}{0}$ & $\begin{array}{l}\text { Fomento trabajo } \\
\text { colaborativo } \\
\text { Fomento de habilidades } \\
\text { de comunicación }\end{array}$ & $\begin{array}{l}\text { Fomento del trabajo } \\
\text { colaborativo } \\
\text { Fomento de habilidades de } \\
\text { comunicación } \\
\text { Fomento del pensamiento } \\
\text { crítico } \\
\text { Desarrollo de habilidades } \\
\text { tecnológicas }\end{array}$ \\
\hline
\end{tabular}

\section{Resultados}

¿Se han conseguido los objetivos que se perseguían al transformar radicalmente el PI para adaptarse a la nueva situación derivada de la pandemia? Al tratarse del primer año que se realiza únicamente podemos hacer referencia a los resultados obtenidos en esta primera edición. De un total de 32 estudiantes, 23 respondieron a un cuestionario sobre diferentes aspectos relativos al PI virtual, esto es, un $72 \%$ de los participantes. Este cuestionario constaba de varios apartados en los que se preguntaba a los participantes por diferentes aspectos del encuentro.

En términos generales y como puede observarse en el cuadro 2 , los estudiantes muestran un elevado grado de satisfacción con el PI (una puntación de 4,38 sobre cinco), así como con su estructura. También coinciden en que el PI puede abrirles nuevas oportunidades profesionales. En términos generales se trata de unos resultados muy satisfactorios, sobre todo teniendo en cuenta que era la primera vez que se realizaba el encuentro virtual y que su dinámica había cambiado por completo.

\section{Tabla 2}

Valoración General del PI

\begin{tabular}{|l|c|}
\cline { 2 - 2 } \multicolumn{1}{c|}{} & $\begin{array}{c}\text { Puntuación } \\
\text { (sobre 5) }\end{array}$ \\
\hline $\begin{array}{l}\text { ¿Cuál es su grado de satisfacción con la } \\
\text { duración de la fase preparatoria del PI (¿25 de } \\
\text { enero - 14 de marzo? }\end{array}$ & 4,23 \\
\hline $\begin{array}{l}\text { ¿En qué medida está satisfecho con la duración } \\
\text { de las jornadas de PI (15-17 de marzo)? }\end{array}$ & 4,35 \\
\hline $\begin{array}{l}\text { El PI y la experiencia internacional me } \\
\text { permitirá mejorar mis oportunidades en el } \\
\text { mercado laboral }\end{array}$ & 4,1 \\
\hline Calificación Global del PI & 4,38 \\
\hline
\end{tabular}

En términos generales, y como queda recogido en la tabla 3, los estudiantes mostraron interés en participar en el PI debido a la posibilidad que ofrecía de trabajar en un entorno internacional. Este fue el principal factor que impulsó su participación (para un 95\% de los encuestados). En segundo y tercer lugar fueron la práctica de lengua extranjera y el propio tema del PI, esto es, profundizar en sus conocimientos sobre el mundo financiero, los que motivaron su interés por participar en el PI. Por el contrario, el motivo menos importante fue la obtención de créditos ETCS (para un $26 \%$ de los estudiantes no fue nada determinante). Hay que recordar que muchas de las universidades que asisten al PI no reconocen con créditos a sus estudiantes la participación en el mismo, lo que explicaría este resultado.

\section{Tabla 3}

¿Qué motivó su decisión de participar en el PI?

\begin{tabular}{|l|c|c|c|c|c|c|}
\cline { 2 - 7 } \multicolumn{1}{l|}{} & MDA & DA & N & DES & $\begin{array}{c}\text { M } \\
\text { DES }\end{array}$ & NS \\
\hline $\begin{array}{l}\text { Reconocimiento } \\
\text { de créditos } \\
\text { ECTS }\end{array}$ & $22 \%$ & $39 \%$ & $4 \%$ & $9 \%$ & $26 \%$ & $0 \%$ \\
\hline El tema del PI & $35 \%$ & $43 \%$ & $22 \%$ & $0 \%$ & $0 \%$ & $0 \%$ \\
\hline $\begin{array}{l}\text { Práctica de } \\
\text { lengua } \\
\text { extranjera } \\
\text { (inglés) }\end{array}$ & $39 \%$ & $48 \%$ & $4 \%$ & $4 \%$ & $0 \%$ & $4 \%$ \\
\hline $\begin{array}{l}\text { Experiencia } \\
\text { internacional }\end{array}$ & $65 \%$ & $30 \%$ & $4 \%$ & $0 \%$ & $0 \%$ & $0 \%$ \\
\hline $\begin{array}{l}\text { Mejora de las } \\
\text { habilidades } \\
\text { "virtuales" }\end{array}$ & $9 \%$ & $35 \%$ & $43 \%$ & $9 \%$ & $0 \%$ & $4 \%$ \\
\hline $\begin{array}{l}\text { Futuro } \\
\text { Profesional }\end{array}$ & $17 \%$ & $52 \%$ & $30 \%$ & $0 \%$ & $0 \%$ & $0 \%$ \\
\hline $\begin{array}{l}\text { Oportunidades } \\
\text { para establecer } \\
\text { contactos }\end{array}$ & $26 \%$ & $48 \%$ & $17 \%$ & $4 \%$ & $0 \%$ & $4 \%$ \\
\hline
\end{tabular}

Nota: MDA (Muy de acuerdo), DA (de acuerdo), N (ni de acuerdo ni en desacuerdo), DES (en desacuerdo), MDES (muy en desacuerdo), NS (no sabe no contesta)

¿Se han cumplido los objetivos que se perseguían con el PI? Como se desprende de la tabla 4 la respuesta es afirmativa. Aparte de las habilidades académicas obtenidas (un 96\% de los estudiantes están de acuerdo o muy de acuerdo en que las han obtenido), cabría destacar la satisfacción con las habilidades de trabajo en equipo (96\%) y de cooperación en un entorno multicultural (91\%). La adquisición de habilidades virtuales es la que obtiene una valoración más baja (es en la única en la que un $4 \%$ está muy en desacuerdo), quizás porque el último año nos ha obligado a todos a actualizarnos en este sentido. Desde esta perspectiva los estudiantes consideran que participar en el PI no las ha mejorado sustancialmente (porque ya las habían desarrollado previamente).

\section{Tabla 4}

Tras haber finalizado el PI cree que ha obtenido habilidades

\begin{tabular}{|l|c|c|c|c|}
\cline { 2 - 5 } \multicolumn{1}{l|}{} & MDA & DA & N & MDES \\
\hline $\begin{array}{l}\text { Académicas, relacionadas } \\
\text { con el contenido del PI }\end{array}$ & $43 \%$ & $52 \%$ & $4 \%$ & $0 \%$ \\
\hline $\begin{array}{l}\text { De colaborar en un } \\
\text { equipo internacional } \\
\text { virtual }\end{array}$ & $48 \%$ & $48 \%$ & $4 \%$ & $0 \%$ \\
\hline $\begin{array}{l}\text { De cooperar en entorno } \\
\text { multicultural }\end{array}$ & $43 \%$ & $48 \%$ & $9 \%$ & $0 \%$ \\
\hline $\begin{array}{l}\text { De construir una red } \\
\text { internacional de contactos }\end{array}$ & $30 \%$ & $52 \%$ & $17 \%$ & $0 \%$ \\
\hline De dominio del inglés & $35 \%$ & $52 \%$ & $13 \%$ & $0 \%$ \\
\hline Habilidades virtuales & $13 \%$ & $61 \%$ & $22 \%$ & $4 \%$ \\
\hline
\end{tabular}

Nota: MDA (Muy de acuerdo), DA (de acuerdo), N (ni de acuerdo ni en desacuerdo), MDES (muy en desacuerdo) 
Por último y para identificar las fortalezas y debilidades del PI, se les preguntó sobre diferentes aspectos de este a los estudiantes. En la tabla 5 se presentan los resultados, ordenados de mayor a menor satisfacción (suma de las columnas muy satisfecho y satisfecho). El claro triunfador del PI fue el juego de escape virtual, seguido por la capacidad y experiencia de los profesores y el propio formato del encuentro. Todos los estudiantes reconocen la labor de los profesores que les tutelan en el pre PI y durante los días del encuentro.

Donde habría que mejorar de cara a futuros encuentros sería en las conferencias ofrecidas a los estudiantes. En este sentido resulta necesario señalar que es muy difícil conseguir que todas las clases magistrales les resulten interesantes, lo que explicaría este resultado. Sin embargo, como punto positivo, el formato introduce la flexibilidad de poder realizar varias conferencias desde diferentes partes del mundo, a lo largo de los 3 días del encuentro virtual, lo que no era posible cuando el encuentro era físico.

\section{Tabla 5}

¿Cuán satisfecho se encuentra con los siguientes aspectos del PI?

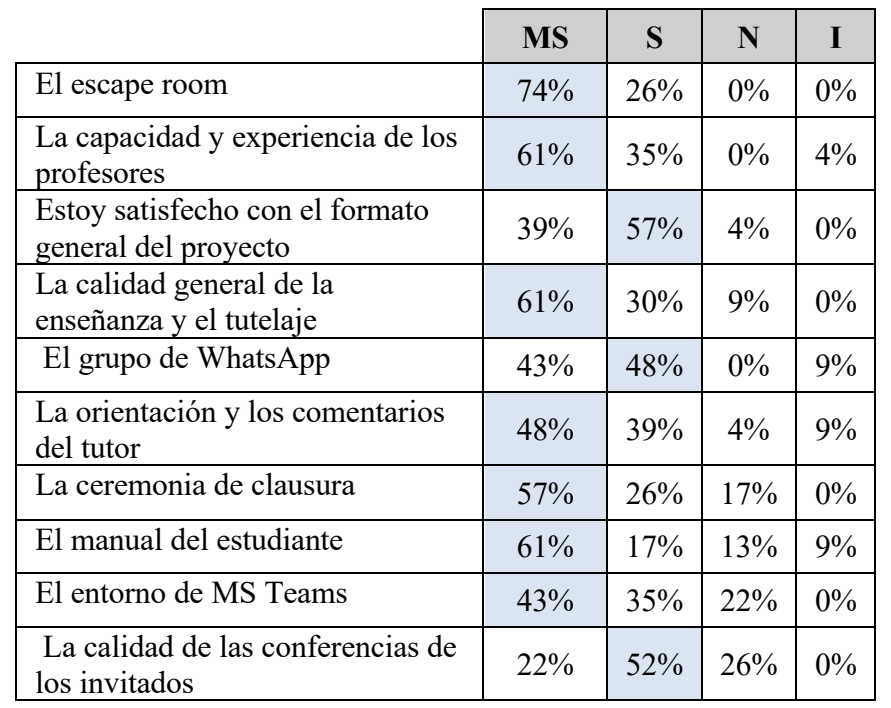

Nota: MS (muy satisfecho), S (satisfecho), N (ni satisfecho ni insatisfecho), I (insatisfecho).

\section{CONCLUSIONES}

Las crisis de cualquier tipo pueden suponer importantes oportunidades. La situación derivada del estallido de la pandemia nos obligó a todos a adaptarnos a una situación extrema a la que nunca nos habíamos enfrentado, ni siquiera imaginado. En el ámbito de la enseñanza nos vimos obligados de la noche a la mañana a reinventarnos. En este contexto un grupo de profesores de diferentes universidades europeas vieron la oportunidad de adaptar un modelo de encuentro que llevaba celebrándose con éxito durante más de un lustro para seguir formando a estudiantes en competencias que les permitieran incorporarse más fácilmente al mercado laboral.

La transformación del PI en un encuentro virtual permitió seguir profundizando en el fomento del trabajo autónomo y colaborativo en un entorno internacional, utilizando para ello un modelo de aprendizaje basado en proyectos. Uno de los participantes del PI virtual destacó del mismo "The opportunity and the idea behind it of providing a multicultural and multiperspective environment, and the last 3 days were so nice working on the interrelated topics". Aunque quizás sea demasiado pronto para extraer conclusiones consistentes, pues sólo se ha celebrado en una ocasión, los datos permiten aventurar que se está trabajando en la dirección adecuada.

Además, la experiencia demuestra que este tipo de aprendizaje basado en proyectos permite a los estudiantes desarrollar múltiples habilidades, que se ven potenciadas cuando se tiene lugar en un ambiente internacional. Sería recomendable iniciar proyectos similares en otras áreas de conocimiento, facultades y escuelas técnicas nacionales y europeas, dados los buenos resultados obtenidos. De cara al próximo curso académico, las autoridades europeas han puesto ya en marcha un nuevo programa Erasmus, "Blended PI", bajo el cual los estudiantes podrán solicitar becas para participar en movilidades de corta duración, lo que encaja perfectamente con el espíritu del PI. La idea es, cuando decaigan definitivamente las restricciones que impiden la movilidad de docentes y estudiantes, combinar el PI virtual con el encuentro físico, manteniendo lo aprendido durante este curso. De esta forma la fase preparatoria continuaría con el mismo diseño puesto en marcha en el curso académico 2020-21. Durante la semana del encuentro se mantendría también la nueva organización y se podría combinar el encuentro físico (para aquellos que quisieran viajar que además contarían con la financiación de la UE), con el encuentro virtual.

\section{AGRADECIMIENTOS}

Este programa no podría haberse llevado a cabo sin el trabajo conjunto de los profesores del PI virtual Petra HogendoornSchweighofer, Luc Salemans y Twan Franken (Inholland University of Applied Sciences), Jana Kotěšovcová, y Jan Mertl (University of Finance and Administration in Prague), Ana Yetano Universidad de Zaragoza), Sven d'Hondt (University College Ghent), Joachim Vogt (Heilbronn University of Applied Sciences), y Monika Foltyn-Zarychta y Joanna Błach (University of Economics de Katowice)

\section{REFERENCIAS}

Fernández Batanero, J.M. (2004). “La transversalidad curricular en el contexto universitario: un puente entre aprendizaje académico y el natural". Revista Fuentes, 5. Recuperado https://revistascientificas.us.es/index.php/fuentes/article/ view/240

Fernández Sánchez, P. y Frank. E. (2019). "Fomentando el trabajo autónomo y cooperativo en un contexto de cooperación internacional: VI Programa Intensivo sobre el futuro de la banca y las finanzas". Actas del V Congreso Internacional sobre Aprendizaje, Innovación y Cooperación. CINAIC 2019. doi: 10.26754/CINAIC.2019.0027

García-Varcálcel Muñoz-Repiso, A. y Basilotta Gómez-Pablos, V. (2017). "Aprendizaje basado en proyectos (ABP): evaluación desde la perspectiva de alumnos de Educación Primaria". Revista de Investigación Educativa, 35(1), 113-131. doi: http://dx.doi.org/10.6018/rie.35.1.246811 
Meyer, E. (2014). "The Culture Map, decoding how people think, lead, and get things done across cultures". Public Affairs. ISBN 978-1-61039-276-1

Van den Bergh, V., Mortermans, D., Spooren, P., Van Petegem, P., Gijbels, D., Vanthournout, G. (2006). "New assessment modes within project-based education - the stakeholders". Studies in Educational Evaluation, 32, 345-368.

https://doi.org/10.1016/j.stueduc.2006.10.005 